\title{
Nephrogenic systemic fibrosis: a new concern for rheumatologists
}

\author{
Jonathan Kay
}

Between 1997 and 2000, 8 of 265 patients who had undergone renal transplantation at a hospital in San Diego, CA, USA, developed joint contractures, brawny induration and hyperpigmentation of the skin on their extremities. Examination of skin biopsies from these patients identified fibrosis and elastosis, often with mucin deposition. Immunohistochemical stains revealed dermal spindle cells, coexpressing CD34, CD45RO and type I procollagen, and histiocytic cells and dermal dendrocytes, expressing CD68 and factor XIIla. These clinical and histological changes were deemed signs of a new disease that was named 'nephrogenic fibrosing dermopathy'. Following the identification of visceral involvement, however, this condition was renamed 'nephrogenic systemic fibrosis' (NSF). Since its initial recognition, the number of cases of NSF worldwide has increased tremendously-all of which have occurred in individuals with chronic kidney disease (CKD). Owing to their experience of caring for patients with scleroderma and other systemic fibrosing disorders, rheumatologists are ideally suited to diagnose and treat patients with NSF.

Careful clinical observation led to the recognition of the probable cause of NSF (Grobner T [2006] Nephrol Dial Transplant 21: 1104-1108). Thomas Grobner, an Austrian nephrologist, observed a correlation between gadoliniumenhanced magnetic resonance angiography and the development of NSF in patients with severe CKD, and postulated that gadolinium exposure was causing the fibrosis. Subsequently, our group found that, among 90 patients with stage 5 CKD, $30 \%$ of those who had previously undergone gadolinium-enhanced imaging studies developed the cutaneous changes seen in NSF (Todd DJ et al. [2007] Arthritis Rheum 56: 34333441). Gadolinium has been detected in skin and other tissues obtained from patients with NSF, further supporting a causal association. In light of this, 'NSF' should be renamed more precisely as 'gadolinium-associated systemic fibrosis.'
Owing to their

experience

of caring for

patients with

scleroderma

and other

systemic

fibrosing

disorders,

rheuma-

tologists are

ideally suited to

diagnose and

treat patients

with NSF

J Kay is an Associate Clinical Professor of Medicine at Harvard Medical School,

Boston, MA, USA.

Competing interests

The author declared no

competing interests.

www.nature.com/clinicalpractice doi:10.1038/ncprheum0887
Based on experience with other environmentally induced cutaneous fibrosing syndromes, such as Spanish toxic oil syndrome and eosinophilia myalgia syndrome, the immediate solution would seem to be to eliminate the toxin in question. However, gadolinium-containing contrast agents are a very useful class of diagnostic drugs that would be difficult to remove from use in all patients.

The majority of NSF cases have been diagnosed in individuals with creatinine clearance rates below $30 \mathrm{ml} / \mathrm{min} / 1.73 \mathrm{~m}^{2}$; accordingly, many institutions have established policies to limit or prohibit the use of gadoliniumcontaining contrast agents in this subgroup of patients. No study has yet been performed, however, to assess the prevalence of NSF in patients with different stages of CKD who have been exposed to gadolinium-containing contrast agents. Thus, this practice might not prevent the development of NSF in individuals with less-severe impairment of renal function.

Increased awareness of this clinical diagnosis will lead to appropriate recognition of cases. So far, however, few therapies have had any effectiveness. Treatment with topical and oral steroids, immunosuppressive drugs and plasmapheresis has been attempted, but without clinically significant improvement. Mildly decreased skin thickening and improved joint mobility has been reported in several patients treated with extracorporeal photopheresis. We have observed notable clinical and histological improvements in skin fibrosis and joint contractures in two patients with NSF who were treated with imatinib mesylate (Kay J and High WA [2008] Arthritis Rheum 58: 2543-2548). A clinical trial of this tyrosine kinase inhibitor in additional patients with NSF is ongoing. Lessons learned from the diagnosis and treatment of patients with NSF will not only benefit these individuals, but also help us to advance our understanding of scleroderma and other fibrosing disorders. 